\title{
全基因组关联研究现状
}

韩建文, 张学军

安徽医科大学皮肤病学研究所, 合肥 230032

摘要: 在过去的 5 年中, 全基因组关联研究(Genome-wide association study, GWAS)方法已被证明是研究复杂疾 病和性状遗传易感变异的一种有效手段。目前, 各国科学家在多种复杂疾病和性状中开展了大量的 GWAS, 对 肿瘤、糖尿病、心脏病、神经精神疾病、自身免疫及免疫相关疾病等复杂疾病以及一些常见性状(如身高、体 重、血脂、色素等)的遗传易感基因研究取得了重大成果。截止到 2010 年 9 月 11 日, 运用 GWAS 开展了对近 200 种复杂疾病/性状的研究, 发现了 3000 多个疾病相关的遗传变异。文章就 GWAS 的发展及其在复杂疾病/ 性状中的应用做一综述。

关键词：全基因组关联研究; 复杂疾病; 性状; 易感基因

\section{Current status of genome-wide association study}

\author{
HAN Jian-Wen, ZHANG Xue-Jun \\ Institute of Dermatology of Anhui Medical University, Hefei 230032, China
}

\begin{abstract}
In past five years, genome-wide association study (GWAS) has proven to be a powerful and efficient study design in identifying genetic variants associated with complex diseases and traits. Many studies have been performed to explore the genetic basis of various complex diseases and traits. Genetic breakthroughs have been achieved in complex diseases and traits, such as oncology, diabetes, cardiology, psychiatric disorders, autoimmune and immune related diseases, height, weight, blood lipid, and pigmentation. Up to September $11^{\text {th }} 2010$, nearly 200 complex diseases and traits have been investigated by this approach, and more than 3000 diseases and/or traits related variants have been identified. In this review, we present an overview of GWAS in complex diseases and traits.
\end{abstract}

Keywords: genome-wide association; complex diseases; traits; susceptibility gene

复杂疾病/性状是由多个微效基因与环境因素 共同作用所致, 具有明显的遗传异质性、表型复杂 性及种族差异性等特征。常见的复杂疾病/性状包括 肿瘤、糖尿病、心脏病、神经精神疾病、自身免疫 及免疫相关疾病、眼科疾病、感染性疾病、消化系 统疾病等严重影响人类身心健康的常见疾病, 以及 身高、体重、血脂、色素等性状。遗传因素在复杂 疾病/性状的发生中起到重要作用。2005 年以来, 随
着“人类基因组计划(Human genome project, HGP)” 和“人类基因组单体型图计划(The international haplotype map project, HapMap)”的相继完成, 以及高通 量基因分型技术的飞速发展, 全基因组关联研究 (Genome-wide association study, GWAS)方法被广泛 应用到多种复杂疾病/性状的遗传学研究中, 并取得 了一系列研究成果。本文就全基因组关联研究的发 展及其在复杂疾病/性状中的应用做一综述。 


\section{GWAS的形成}

\subsection{GWAS的研究背景}

在关联研究方法提出以前, 人们主要利用连锁 研究方法开展对复杂疾病/性状遗传易感性研究, 并 且取得一定成绩，发现了一些疾病和性状的易感基 因。但由于复杂疾病/性状具有明显的遗传异质性、 表型复杂性等特点，使得以家系为基础的“全基因组 连锁研究”的应用受到了限制 $\frac{11 \sim 3]}{1}$ 。1996 年, Risch和 Merikangas的研究显示在常见复杂疾病的遗传学研 究中关联研究较连锁研究有更高的效力, 并提出全 基因组关联研究的概念 ${ }^{[4]}$ 。关联研究是基于“常见疾 病, 常见变异”(Common disease, common variant)的 假设, 其基本原理是: 在一定人群中选择病例组和 对照组, 比较全基因组范围内所有单核苷酸多态性 (Single nucleotide polymorphism, SNP)位点的等位基 因或者基因型频率在病例组与对照组间的差异。如 果某个SNP位点的等位基因或基因型在病例组中出 现的频率明显高于或低于对照组, 则认为该位点与 疾病间存在关联性。之后根据该位点在基因组中的 位置和连锁不平衡关系推测可能的疾病易感基因。 比如某个SNP位点的等位基因 C在糖尿病患者中的 频率是 0.355 , 而在对照中的频率是 0.123 , 经过统 计分析发现差异有显著性, 可以说此基因位点与疾 病存在关联性。GWAS本质上也属于关联研究。

随着DNA测序技术自动化程度和基因分型通量 的日益提高，全世界多个国家的科研机构通过合作， 相继完成了 HGP和HapMap计划, 使人类对于生命本 源的认识大大向前推进了一步。2003 年HGP计划的 完成为阐明人类基因组序列, 解读生命遗传密码, 提供了研究基因结构和功能的基础。HapMap计划描 述和记录在不同人群中的基因组中常见遗传变异的 等位基因和基因型频率及单倍型形式, 分别在 2005 年、2007 年和 2009 年公布了第 I 、II 、III期(Phase I , II , III)的数据。目前该计划已发现了人类基因组 中 1000 多万个常见SNPs, 构建了人类基因组差异 的公众数据库(www.hapmap.org)。HapMap计划为研 究者提供了将遗传多态位点(高密度的遗传变异图 谱)与特定疾病/性状风险联系的相关信息, 使得 GWAS的应用成为可能, 从根本上改变了基因研究
的“蓝图”, 进而为疾病的预防, 诊断和治疗提供了 新的方法 ${ }^{[5]}$ 。

如果说HGP和HapMap计划使GWAS 在理论上 成为可能，那么近年来飞速发展的基因分型技术、 不断完善的统计学方法和统计分析软件则为 GWAS 的开展提供了技术上的保障。基于HapMap的人类全 基因组SNPs检测芯片问世后, 研究者能够同时对每 一个体的数十万到一百万个SNPs进行检测 ${ }^{[6 \sim 8]}$; 高 效统计分析软件的出现使得处理海量分型数据的难 题也迎刃而解 ${ }^{[9]}$ 。

\subsection{GWAS的设计和过程}

目前的 GWAS 多采用两个阶段的设计: 首先采 用覆盖整个基因组的高通量 SNPs 分型芯片对一批 样本进行扫描, 然后篮选出最显著的 SNPs( 如 $P<10^{-7}$ )供第二阶段进行扩大样本验证。GWAS 两 阶段研究设计减少了基因分型的工作量和花费，同 时通过重复实验降低了研究的假阳性率。GWAS 的 整体过程比较复杂, 其大致流程如下: (1)经过处理 的 DNA 样品与高通量的 SNP 分型芯片进行杂交; (2)通过特定的扫描仪对芯片进行扫描, 将每个样品 所有的 SNP 分型信息以数字形式储存于计算机中; (3)对原始数据进行质控, 检测分型样本和位点的得 率(call rate)、病例对照的匹配程度、人群结构的分层 情况等; (4)对经过各种严格质控的数据进行关联分析; (5)根据关联分析结果, 综合考虑基因功能多方面因素 后, 篮选出最有意义的一批 SNP 位点; (6)根据需要验 证 SNP 的数量选择合适通量的基因分型技术在独立样 本中进行验证; (7)合并分析 GWAS 两阶段数据。

目前应用于 GWAS 的全基因组分型的主流技术 平台来自 Illumina 公司和 Affymetrix 公司。每张基 因分型芯片上包含了成千上万的 SNPs 检测探针, 而且随着检测技术的不断完善, 芯片的分型通量还 在不断增加, 从最初含 1 万 SNPs 探针的 Affymetric $10 \mathrm{~K}$ 基因分型芯片到目前包含数十万甚至一百万探 针的 Affymetric $500 \mathrm{~K}$ 、Affymetric 6.0、Illumina 300 $\mathrm{K} 、$ Illumina 610-quad、Illumina $1 \mathrm{M}$ 等高密度分型芯 片; 还有部分芯片整合了拷贝数变异(Copy number variations, CNVs)探针，如：Illumina 370、Illumina 610-quad 以及 Affymetric 6.0 等, 研究者可以同时对 人类基因组常见变异 SNPs 和 CNVs 进行分析。 
随着分型技术不断完善，对每个SNP的分型成 本显著降低, 从而使GWAS已经被广泛应用于多种 复杂疾病的研究, 使人类对复杂疾病/性状易感基因 研究现状得到彻底的改变 ${ }^{[6-8]}$ 。2005 年, Science 杂志 首次报道了利用商业化基因芯片进行复杂疾病的 GWAS。Klein等 ${ }^{[10]}$ 利用Affymetrix 100K基因芯片对 年龄相关性视网膜黄斑变性进行了 GWAS, 并成功 发现一个与疾病密切相关的基因 $C F H$ 。随后, 一系 列复杂疾病/性状的GWAS在全世界不同研究小组中 相继开展, 并取得很大的成功。2007 年, 威康信托 基金会病例对照研究协会 (Wellcome Trust Case Control Consortium, WTCCC)利用对 7 种常见复杂疾 病(双向性情感障碍、冠心病、克隆病、高血压、类 风湿性关节炎、1 型糖尿病、2 型糖尿病)共计 17000 个样本进行了大规模的GWAS, 发现了上述疾病的 众多易感基因位点。随后, 该研究发现的众多疾病 相关位点被其它研究团队所证实。该项研究是迄今 为止规模最大的GWAS, 对以后的复杂疾病/性状的 GWAS的开展起到了典范作用 ${ }^{[11]}$ 。

\section{2 复杂疾病的GWAS}

在过去的 5 年里, 各国科学家开展了对不同疾 病的GWAS, 涉及到肿瘤、心血管系统疾病、内分泌 系统疾病、胃肠道疾病、肝脏疾病、眼科疾病、神 经精神类疾病、风湿病、皮肤病以及感染性疾病等 领域, 并取得了重大成果。GWAS在复杂疾病遗传学 研究中取得的成果加深了我们对这些复杂疾病遗传 学基础的认识, 为后续对其发病机制研究提供启示, 也为将来的的基因诊断和个体化治疗奠定了理论基 础。截止到 2010 年 9 月 11 日, 已经对近 200 种复 杂疾病和性状开展 600 多项GWAS, 发现 3000 多个 与疾病/性状相关SNPs, 确定的疾病/性状相关的易 感基因或位点达 700 多个, 发表论文数逐年递增(图 1)。美国癌症研究所(National Cancer Institute, NCI)、 美国国家人类基因组研究所(National Human Genome Research Institute, NHGRI)对近年来发表的 GWAS文献进行总结汇总, 公布在其网站上, 并定 期更新，供世界各地研究人员免费查询

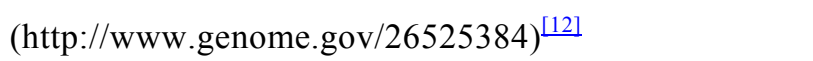
病为例讨论GWAS对复杂疾病的意义。

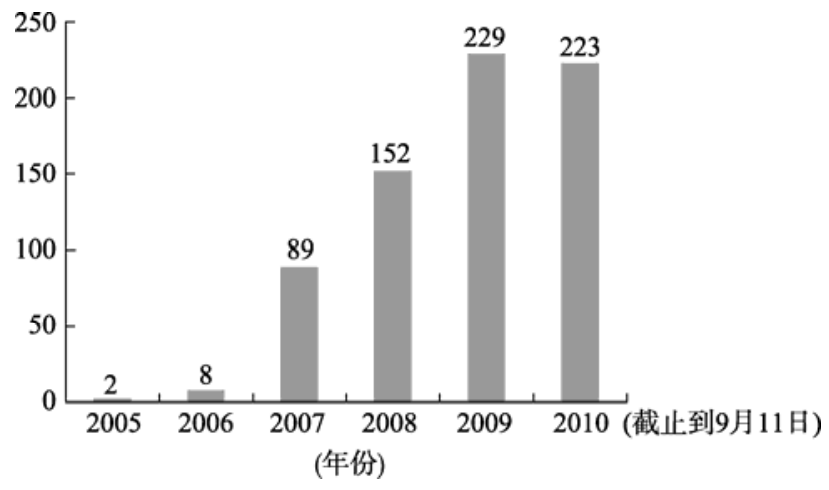

图 12005 2010 年 GWAS 文章数量走势图

\subsection{1 型糖尿病}

1 型糖尿病是一种复杂疾病, 其发病与遗传及 环境因素有关。在GWAS应用之前通过候选基因和 定位的方法确定的 1 型糖尿病发病相关的易感基因 有 6 个, 但这些易感基因主要在家族聚集的患者发 病中起作用, 尚不能完全解释散发病例的易感性。 Todd等 ${ }^{[13]}$ 利用WTCCC的数据 ${ }^{[11]}$, 从提示与 1 型糖 尿病关联的SNP中选择 11 个SNP, 在另外独立的样 本中进行了验证。最后证实了 12q13.2(ERBB3)、 12q24.13(C12orf30、SH2B3/LNK)、16p13.13(KIAA0350) 和 $18 \mathrm{p} 11.21(P T P N 2)$ 与 1 型糖尿病发病密切相关。与 此同时, Hakonarson等 ${ }^{[14]}$ 利用Illumina $550 \mathrm{~K}$ 基因芯 片进行的 1 型糖尿病 GWAS不仅也发现位于 16p13.13(KIAA0350)与 1 型糖尿病的易感性相关联, 同时还证实 2 个以往通过定位方法发现的 1 型糖尿 病易感基因INS、PTPN22。此外，在对KIAA0350 基 因的功能研究中发现携带危险等位基因的个体, NK 细胞中KIAA0350 表达量上调, 其与 1 型糖尿病的自 身免疫发病机制有关。目前国际上包括WTCCC在内 的多个研究小组已运用GWAS对 1 型糖尿病进行了 8 项大规模的GWAS, 发现了 40 多个与 1 型糖尿病 发病相关的易感基因。其中, 被 3 项以上研究证实 的、有比较肯定结论的 1 型糖尿病易感基因有 12 个, 如 $1 \mathrm{p} 13.2(P T P N 22) 、 2 \mathrm{q} 24.2(I F I H 1) 、$ 2q33.2(CTLA4)、6p21.32(MHC)、6q15(BACH2)、 10p15.1(PRKCQ-IL2RA)、11p15.5(INS)、12p13.31、 12q13.2(ERBB3)、12q24.13(C12orf30、SH2B3/LNK)、 16p13.13(KIAA0350)、18p11.21(PTPN2) ${ }^{[15,16]}$ 。

\subsection{2 型糖尿病}

2 型糖尿病是一种常见的复杂疾病, 占据糖尿 
病群体的大多数 (95\%), 其发病也与易感基因密切 相关。2006 年, 冰岛的一个遗传研究机构deCODE 采用关联研究方法发现了 TCF $7 L 2$ 基因与 2 型糖尿 病的发病密切相关 ${ }^{[17]}$ 。此后, 该结果至少在 20 个不 同人种和遗传背景的人群中得到验证, 结果的一致 性表明TCF7L2 基因为普遍存在的 2 型糖尿病易感 基因 ${ }^{[18]}$ 。2007 年, 对 2 型糖尿病的易感基因研究取 得了重大进展, 由威康信托基金会病例-对照研究协 会、美国糖尿病遗传研究协会、芬兰国家非胰岛素 依赖型糖尿病遗传研究组织及冰岛的deCODE遗传 研究团体进行了一系列 2 型糖尿病的GWAS ${ }^{[19-22]}$, 并进行合作和数据共享, 共新发现 8 个 2 型糖尿病 的易感位点, 即 $C D K N 2 A / C D K N 2 B 、 C D K A L 1$ 、 IGF2BP2、AHI1-LOC441171、EXT2-ALX4、IDE-KIF11$H H E X$ 和 SLC $30 A 8$ 。该些研究的成功, 显示了不同研 究机构之间科研协作和数据共享在大规模GWAS中 的重要性。通过协作, 使得应用于GWAS的样本量达 到 3 万多例。正是因为具备了如此大的样本量, 研 究者们才能够发现遗传效应较低(OR值为 1.1 1.2)的 易感基因位点。除了这些发现, 他们还成功验证了 一些之前已知的与 2 型糖尿病相关联的基因, 如 TCF7L2、PPARG、KCNJ11。迄今为止, 各国科学家 已经应用GWAS先后开展了 20 余项搜寻 2 型糖尿病 的易感基因研究, 共发现 40 余个 2 型糖尿病的易感 基因/位点 ${ }^{[23]}$ 。

总之, 这些易感基因的发现对进一步理解 1、2 型糖尿病的遗传学基础和发病机制具有重要的意义, 不仅对今后糖尿病发病机制的研究奠定了基础, 而 且为基因诊断、靶向治疗带来契机 $\stackrel{[24]}{2}$

\section{3 性状的GWAS}

人类的一些常见性状，如身高、体重、色素、 血脂等也受到遗传和环境共同影响, 呈多基因遗传 模式, 而且在不同人种间、或者同一人群中存在差 异。有些性状是种族特异性的, 比如不同种族肤色 的差异, 但是在相同种族人群中也有差异存在。在 GWAS的广泛应用之前仅有很少的性状相关基因被 证实。近年来, 许多性状的GWAS相继开展, 目前进 行的性状GWAS已经超过 100 多项, 涉及身高

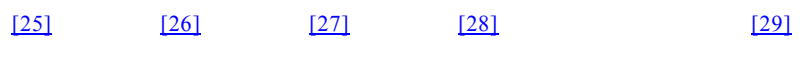
骨质疏松 ${ }^{[30]}$ 、肺功能 ${ }^{[311}$ 及多种血清生物标记 ${ }^{[32]}$ 等常
见性状。这些性状GWAS的大多数在研究设计之初 并不是为了研究性状的遗传学基础而启动的, 而是 在进行其他疾病GWAS的同时, 收集病例和对照样 本相应的性状信息, 最后深入分析GWAS数据进行 研究性状的相关基因。GWAS的广泛应用在揭示人 类性状遗传基础的进程中起到了极大的推动作用。

有的性状与复杂疾病的发生有着密切的关系, 例如肥胖与 2 型糖尿病、皮肤色素与皮肤肿瘤、血 脂与冠心病、血清尿酸水平与痛风、骨质疏松与钙 磷代谢及骨折、肺功能与慢性阻塞性肺疾病等。例 如Frayling 等 ${ }^{[33]}$ 进行的 2 型糖尿病GWAS最初发现在 FTO基因与 2 型糖尿病显著关联。但是当以体重指 数(Body mass index, BMI)进行校正以后, FTO基因 变化与 2 型糖尿病不再具有关联性。他们意外地发 现了FTO基因是通过增加肥胖(或BMI)的风险而增 加 2 型糖尿病的发病风险。此后, 多项独立的究结 果都显示 $F T O$ 基因与 $\mathrm{BMI}$ 及肥胖存在关联性 [26,34]。另外, $M C 1 R$ 基因(16q24)最初被确定为色素相 关的基因，与毛发、皮肤色素、雀斑以及皮肤对日 光反应具有关联性 ${ }^{[35]}$, 在随后的研究中又被发现与 黑色素瘤的易感性相关 ${ }^{[36]}$ 。另外一个色素相关的基 因TYR 在 Jin等 ${ }^{[37]}$ 的GWAS中被确定为白癜风的易感 基因。Gudbjartsson等 ${ }^{[38]}$ 在几种皮肤肿瘤中研究色素 相关基因的关联性，发现 $A S I P$ 和 TYR 基因与皮肤基 底细胞癌和黑素瘤的易感性相关。因此, 对人类性 状进行相关遗传学研究的意义不仅仅对性状本身, 而且在复杂疾病的研究中也起到重要的作用。

另外, 科学家们还将GWAS应用到疾病治疗的 研究中, 已经有 25 篇相关的研究被报道, 并发现了 与药物治疗反应以及药物副作用相关的一些基 因 ${ }^{[39,40]}$ 。这些研究涉及到抗抑有险、精神疾病药物、 丙型肝炎等治疗反应，以及肝损害以及其他副作用， 发现 140 多个与药物治疗反应和/或副作用相关的基 因。但是其中一些研究的样本量较小, 还有待更多 的研究来验证这些研究结果, 药物反应的相关基因 的研究为将来根据患者遗传信息的差异进行个体化 治疗的尝试积累了经验。

\section{4 中国GWAS的发展}

虽然复杂疾病/性状的GWAS在中国起步较晚, 但是我国科学家通过不解努力, 近三年来取得一系 
列重大研究成果, 逐渐赶上了国际GWAS水平。2008 年, 贺林院士研究组与加迪夫大学、牛津大学、剑 桥大学、斯坦福大学等多家学术机构合作, 开展对 精神分裂症的GWAS，发现ZNF804A基因与精神分 裂症的易感性相关 ${ }^{[41]}$ 。2009 年, 笔者所在实验室完 成了国内首个中国人银屑病的GWAS，涉及的样本 量达到 6860 例银屏病患者和 8472 正常对照, 发现 了位于染色体 $1 \mathrm{q} 21$ 上的编码晚期角质化包膜(Late cornified envelope, LCE)基因簇与银屑病的遗传关联 性, 提示在银屑病的发生、发展过程中一种非免疫 机制一一表皮角质形成细胞的终末分化在疾病发病 机制中的重要作用, 其研究成果在欧洲人种的研究 中也得到验证 ${ }^{[42]}$ 。同时, 该研究还证实了既往报道 的银屑病易感基因 $M H C$ 和 IL $2 \mathrm{~B}$ [43]。

此后，我们又陆续完成对系统性红斑狼疮、麻 风、白癜风的GWAS。通过对中国汉族人系统性红 斑狼疮 (Systemic lupus erythematosus, SLE) 开展 GWAS，发现了 9 个新的SLE易感基因/位点，同时 验证了既往报道的 7 个易感基因/位点, 上述研究结 果证实不同人种间SLE既存在共同的易感基因，也 具有遗传异质性 ${ }^{[44]}$ 。我们新发现的这 9 个SLE易感 基因/位点分别是 $2 \mathrm{p} 22.3$ (RASGRP3)、5q33.1(TNIP1)、 7p12.2(IKZF1)、11q24.3(ETS1)、12q24.32(SLC15A4)、 7q11.23、10q11.22(WDFY4-LRRC18)、11q23.3 和 $16 \mathrm{p} 11.2 ， 5$ 个确定的易感基因 (RASGRP3、TNIP1、 $I K Z F 1 、 E T S 1$ 和SLC15A4)在基因功能上都与抗原呈 递、免疫反应、免疫调节、淋巴细胞活化等过程密 切相关。其中, 3 个新发现的SLE易感基因(TNIP1、 ETS1 和 WDFY4)分别在欧洲人群和另一项亚洲人 群SLE的GWAS中得到验证 ${ }^{[45,46]}$ 。麻风GWAS发现 7 个新的易感基因：CCDC122，C13orf31，NOD2, TNFSF15, HLA-DR, RIPK2 和LRRK2, 其中 4 个基因 (C13orf31、LRRK2、NOD2、RIPK2)与多菌性麻风 的关联性比少菌型的关联性更强, 5 个易感基因位于 NOD2 介导的信号通路上, 提示该信号通路在麻风 发病中具有重要作用, 为揭示疾病发病机制提供了 重要契机 ${ }^{[47]}$ 。同时, 通过对不同复杂疾病开展 GWAS研究时还发现了许多复杂疾病存在共同的易 感基因，提示不同疾病间可能存在共同的发病机 制。如上述麻风GWAS发现的易感基因 CCDC122、 C13orf31、NOD2 和TNFSF15 在既往的研究中显示
与克隆病相关 ${ }^{[48,49]}$, 提示有共同的机制参与两种疾 病的发生。此外，今年我们刚完成的白癜风GWAS 发现在 6q27 区域存在该病重要易感基因(RNASET2、 $F G F R 1 O P$ 和 CCR6 $)^{[50]}$ ，而该区域也曾被报道与克隆 病相关 ${ }^{[49]}$, 其也提示两种疾病在发病中可能有着共 同的机制。

今年, 我国在肿瘤GWAS中也取得喜人的成绩。 曾益新院士开展的鼻咽癌GWAS除证实HLA与鼻咽 癌的关联性外，发现 3 个新的易感基因 TNFRSF19(13q12)、MDS1-EVI1(3q26) 和 CDKN2A$C D K N 2 B(9 \mathrm{p} 21)^{[51]}$ 。贺福初院士和周钢桥教授开展了 乙肝病毒相关肝癌的GWAS, 发现一个疾病相关位 点 $1 \mathrm{p} 36.22$, 位于该区域的 3 个基因 $K I F 1 B 、 U B E 4 B$ 或 $P G D$ 中可能有一个是乙肝病毒相关肝癌的易感基 因 ${ }^{[52]}$ 。王立东教授与我们课题组合作开展的食管癌 GWAS发现两个新的食管癌易感基因, 分别是位于 $10 \mathrm{q} 23$ 的PLCE1 和位于 $20 \mathrm{p} 13$ 的C20orf54, 并通过验 证实验证实这两个基因与赑门癌易感性也显著相 关。此外, 免疫组化研究显示PLCE1 蛋白在食管癌 和贲门癌组织中的表达明显高于正常食管和胃贲门 粘膜组织 ${ }^{[53]}$ 。在今年, 为了更加深入的开展寻找疾 病易感基因研究，我们开展了对前期银屑病GWAS 数据深入分析, 并通过扩大验证样本量等方法以进 一步寻找新的银屑病易感基因。该研究以中国人群 为主，并与美国、德国等多家国际银屑病研究组织 进行合作, 对我们的研究结果在欧美人群中进行了 验证研究, 研究样本共涉及 11605 个患者和 17107 个对照及 254 个核心家系。结果又新发现了 6 个与 银屑病相关的易感基因位点 ${ }^{[54]}$ 。

\section{5 结 语}

总之, GWAS 在复杂疾病/性状的研究取得了令 人鼓舞的成绩, 发现了大量复杂疾病/性状相关的易 感基因/位点; 另外还发现某些疾病存在共同的易感 基因，提示不同疾病可能有着共同的发病机制，这 些研究成果将深化我们对于这些疾病和性状的认 识。近两年我国的 GWAS 赶上国际科学发展步伐, 一系列 GWAS 项目的成功实施和取得的突破性成果 开辟了我国复杂疾病性状 GWAS 新局面, 为我国将 来的复杂疾病/性状研究提供了范例和经验。但是由 于 GWAS 的理论基础是“常见疾病，常见变异”的假 
说，在复杂疾病/性状的易感基因研究中仍存在一些 不足之处，仅适用于疾病相关的常见变异研究，对 于影响复杂疾病/性状的罕见变异和基因组结构变 异不敏感。另外, 目前 GWAS 发现的一些疾病/性状 的相关变异多数位于基因组的非编码区，它们与疾 病相关性可能是通过与真正疾病/性状相关变异的 连锁不平衡而引起的。因此, 鉴定出真正与疾病/性 状相关的易感基因和/或易感变异仍然任重道远; 确 定疾病/性状易感基因的功能以及在疾病/性状发生 发展中的作用仍然需要更加深入的研究，比如进行 精细定位研究寻找疾病/性状相关变异、易感基因的 功能和结构以及转录调节方面的相关研究。因此, GWAS 只提示某些基因与疾病/性状具有关联性, 为 疾病/性状机制的研究提供启示，至于基因如何影响 疾病/性状的发生发展还将是一个更大的挑战。

\section{参考文献(References):}

[1] Göring HH, Terwilliger JD, Blangero J. Large upward bias in estimation of locus-specific effects from genomewide scans. Am J Hum Genet, 2001, 69(6): 1357-1369.[DOI]

[2] Lander E, Kruglyak L. Genetic dissection of complex traits: guidelines for interpreting and reporting linkage results. Nat Genet, 1995, 11(3): 241-247. [DOI]

[3] Sabatti C, Service S, Freimer N. False discovery rate in linkage and association genome screens for complex disorders. Genetics, 2003, 164(2): 829-833. [DOI]

[4] Risch N, Merikangas K. The future of genetic studies of complex human diseases. Science, 1996, 273(5281): 1516-1517. [DOI]

[5] Frazer KA, Ballinger DG, Cox DR, Hinds DA, Stuve LL, Gibbs RA, Belmont JW, Boudreau A, Hardenbol P, Leal SM, Pasternak S, Wheeler DA, Willis TD, Yu F, Yang H, Zeng C, Gao Y, Hu H, Hu W, Li C, Lin W, Liu S, Pan H, Tang X, Wang J, Wang W, Yu J, Zhang B, Zhang Q, Zhao H, Zhou J, Gabriel SB, Barry R, Blumenstiel B, Camargo A, Defelice M, Faggart M, Goyette M, Gupta S, Moore J, Nguyen H, Onofrio RC, Parkin M, Roy J, Stahl E, Winchester E, Ziaugra L, Altshuler D, Shen Y, Yao Z, Huang W, Chu X, He Y, Jin L, Liu Y, Sun W, Wang H, Wang Y, Xiong X, Xu L, Waye MM, Tsui SK, Xue H, Wong JT, Galver LM, Fan JB, Gunderson K, Murray SS, Oliphant AR, Chee MS, Montpetit A, Chagnon F, Ferretti V, Leboeuf M, Olivier JF, Phillips MS, Roumy S, Sallé e C, Verner A, Hudson TJ,
Kwok PY, Cai D, Koboldt DC, Miller RD, Pawlikowska L, Taillon-Miller P, Xiao M, Tsui LC, Mak W, Song YQ, Tam PK, Nakamura Y, Kawaguchi T, Kitamoto T, Morizono T, Nagashima A, Ohnishi Y, Sekine A, Tanaka T, Tsunoda T, Deloukas P, Bird CP, Delgado M, Dermitzakis ET, Gwilliam R, Hunt S, Morrison J, Powell D, Stranger BE, Whittaker P, Bentley DR, Daly MJ, de Bakker PI, Barrett J, Chretien YR, Maller J, McCarroll S, Patterson N, Pe'er I, Price A, Purcell S, Richter DJ, Sabeti P, Saxena R, Schaffner SF, Sham PC, Varilly P, Stein LD, Krishnan L, Smith AV, Tello-Ruiz MK, Thorisson GA, Chakravarti A, Chen PE, Cutler DJ, Kashuk CS, Lin S, Abecasis GR, Guan W, Li Y, Munro HM, Qin ZS, Thomas DJ, McVean G, Auton A, Bottolo L, Cardin N, Eyheramendy S, Freeman C, Marchini J, Myers S, Spencer C, Stephens M, Donnelly P, Cardon LR, Clarke G, Evans DM, Morris AP, Weir BS, Mullikin JC, Sherry ST, Feolo M, Skol A, Zhang H, Matsuda I, Fukushima Y, Macer DR, Suda E, Rotimi CN, Adebamowo CA, Ajayi I, Aniagwu T, Marshall PA, Nkwodimmah C, Royal CD, Leppert MF, Dixon M, Peiffer A, Qiu R, Kent A, Kato K, Niikawa N, Adewole IF, Knoppers BM, Foster MW, Clayton EW, Watkin J, Muzny D, Nazareth L, Sodergren E, Weinstock GM, Yakub I, Birren BW, Wilson RK, Fulton LL, Rogers J, Burton J, Carter NP, Clee CM, Griffiths M, Jones MC, McLay K, Plumb RW, Ross MT, Sims SK, Willey DL, Chen Z, Han H, Kang L, Godbout M, Wallenburg JC, L'Archevê que P, Bellemare G, Saeki K, An D, Fu H, Li Q, Wang Z, Wang R, Holden AL, Brooks LD, McEwen JE, Guyer MS, Wang VO, Peterson JL, Shi M, Spiegel J, Sung LM, Zacharia LF, Collins FS, Kennedy K, Jamieson R, Stewart J. A second generation human haplotype map of over 3.1 million SNPs. Nature, 2007, 449(7164): 851-861. [DOI]

[6] Steemers FJ, Gunderson KL. Whole genome genotyping technologies on the BeadArray platform. Biotechnol $J$, 2007, 2(1): 41-49. [DOI]

[7] Kennedy GC, Matsuzaki H, Dong SL, Liu WM, Huang J, Liu GY, Su X, Cao MQ, Chen WW, Zhang J, Liu WW, Yang G, Di XJ, Ryder T, He ZJ, Surti U, Phillips MS, Boyce-Jacino MT, Fodor SP, Jones KW. Large-scale genotyping of complex DNA. Nat Biotechnol, 2003, 21(10): 1233-1237. [DOI]

[8] Gunderson KL, Steemers FJ, Lee G, Mendoza LG, Chee MS. A genome-wide scalable SNP genotyping assay using microarray technology. Nat Genet, 2005, 37(5): 549-554. 
[DOI]

[9] Purcell S, Neale B, Todd-Brown K, Thomas L, Ferreira MA, Bender D, Maller J, Sklar P, de Bakker PI, Daly MJ, Sham PC. PLINK: a tool set for whole-genome association and population-based linkage analyses. Am J Hum Genet, 2007, 81(3): 559-575. [DOI]

[10] Klein RJ, Zeiss C, Chew EY, Tsai JY, Sackler RS, Haynes C, Henning AK, SanGiovanni JP, Mane SM, Mayne ST, Bracken MB, Ferris FL, Ott J, Barnstable C, Hoh J. Complement factor $\mathrm{H}$ polymorphism in age-related macular degeneration. Science, 2005, 308(5720): 385-389. [DOI]

[11] Burton PR, Clayton DG, Cardon LR, Craddock N, Deloukas P, Duncanson A, Kwiatkowski DP, McCarthy MI, Ouwehand WH, Samani NJ, Todd JA, Donnelly P, Barrett JC, Burton PR, Davison D, Donnelly P, Easton D, Evans D, Leung HT, Marchini JL, Morris AP, Spencer CC, Tobin MD, Cardon LR, Clayton DG, Attwood AP, Boorman JP, Cant B, Everson U, Hussey JM, Jolley JD, Knight AS, Koch K, Meech E, Nutland S, Prowse CV, Stevens HE, Taylor NC, Walters GR, Walker NM, Watkins NA, Winzer T, Todd JA, Ouwehand WH, Jones RW, McArdle WL, Ring SM, Strachan DP, Pembrey M, Breen G, St Clair D, Caesar S, Gordon-Smith K, Jones L, Fraser C, Green EK, Grozeva D, Hamshere ML, Holmans PA, Jones IR, Kirov G, Moskvina V, Nikolov I, O'Donovan MC, Owen MJ, Craddock N, Collier DA, Elkin A, Farmer A, Williamson R, McGuffin P, Young AH, Ferrier IN, Ball SG, Balmforth AJ, Barrett JH, Bishop DT, Iles MM, Maqbool A, Yuldasheva N, Hall AS, Braund PS, Burton PR, Dixon RJ, Mangino M, Suzanne S, Tobin MD, Thompson JR, Samani NJ, Bredin F, Tremelling M, Parkes M, Drummond H, Lees CW, Nimmo ER, Satsangi J, Fisher SA, Forbes A, Lewis CM, Onnie CM, Prescott NJ, Sanderson J, Mathew CG, Barbour J, Mohiuddin MK, Todhunter CE, Mansfield JC, Ahmad T, Cummings FR, Jewell DP, Webster J, Brown MJ, Clayton DG, Lathrop GM, Connell J, Dominczak A, Samani NJ, Marcano CA, Burke B, Dobson R, Gungadoo J, Lee KL, Munroe PB, Newhouse SJ, Onipinla A, Wallace C, Xue M, Caulfield M, Farrall M, Barton A, Bruce IN, Donovan H, Eyre S, Gilbert PD, Hider SL, Hinks AM, John SL, Potter C, Silman AJ, Symmmons DP, Thomson W, Worthington J, Clayton DG, Dunger DB, Nutland S, Stevens HE, Walker NM, Widmer B, Todd JA, Frayling TA, Freathy RM, Lango H, Perry JR, Shields BM, Weedon MN, Hattersley AT, Hitman GA, Walker M, Elliott KS, Groves CJ, Lindgren
CM, Rayner NW, Timpson NJ, Zeggini E, McCarthy MI, Newport M, Sirugo G, Lyons E, Vanberg F, Hill AV, Bradbury LA, Farrar C, Pointon JJ, Wordsworth P, Brown MA, Franklyn JA, Heward JM, Simmonds MJ, Gough SC, Seal S, Stratton MR, Rahman N, Ban M, Goris A, Sawcer SJ, Compston A, Conway D, Jallow M, Newport M, Sirugo G, Rockett KA, Kwiatowski DP, Bumpstead SJ, Chaney A, Downes K, Ghori MJ, Gwilliam R, Hunt SE, Inouye M, Keniry A, King E, McGinnis R, Potter S, Ravindrarajah R, Whittaker P, Widden C, Withers D, Deloukas P, Leung HT, Nutland S, Stevens HE, Walker NM, Todd JA, Easton D, Clayton DG, Burton PR, Tobin MD, Barrett JC, Evans D, Morris AP, Cardon LR, Cardin NJ, Davison D, Ferreira T, Pereira-Gale J, Hallgrimsdottir IB, Howie BN, Marchini JL, Spencer CC, Su Z, Teo YY, Vukcevic D, Donnelly P, Bentley D, Brown MA, Gordon LR, Caulfield M, Clayton DG, Compston A, Craddock N, Deloukas P, Donnelly P, Farrall M, Gough SC, Hall AS, Hattersley AT, Hill AV, Kwiatkowski DP, Mathew C, McCarthy MI, Ouwehand WH, Parkes M, Pembrey M, Rahman N, Samani NJ, Stratton MR, Todd JA, Worthington J. Genome-wide association study of 14,000 cases of seven common diseases and 3,000 shared controls. Nature, 2007, 447(7145): 661-678. [DOI]

[12] Hindorff L, Junkins H, Mehta J, Manolio T. A catalog of published Genome-Wide Association Studies. Available at: www.genome.gov/gwastudies.

[13] Todd JA, Walker NM, Cooper JD, Smyth DJ, Downes K, Plagnol V, Bailey R, Nejentsev S, Field SF, Payne F, Lowe CE, Szeszko JS, Hafler JP, Zeitels L, Yang JH, Vella A, Nutland S, Stevens HE, Schuilenburg H, Coleman G, Maisuria M, Meadows W, Smink LJ, Healy B, Burren OS, Lam AA, Ovington NR, Allen J, Adlem E, Leung HT, Wallace C, Howson JM, Guja C, Ionescu-Tirgoviste C, Simmonds MJ, Heward JM, Gough SC, Dunger DB, Wicker LS, Clayton DG. Robust associations of four new chromosome regions from genome-wide analyses of type 1 diabetes. Nat Genet, 2007, 39(7): 857-864. [DOI]

[14] Hakonarson H, Grant SF, Bradfield JP, Marchand L, Kim CE, Glessner JT, Grabs R, Casalunovo T, Taback SP, Frackelton EC, Lawson ML, Robinson LJ, Skraban R, Lu Y, Chiavacci RM, Stanley CA, Kirsch SE, Rappaport EF, Orange JS, Monos DS, Devoto M, Qu HQ, Polychronakos C. A genome-wide association study identifies KIAA0350 as a type 1 diabetes gene. Nature, 2007, 448(7153): 591-594. 
[DOI]

[15] Concannon P, Rich SS, Nepom GT. Genetics of type 1A diabetes. $N$ Engl J Med, 2009, 360(16): 1646-1654. [DOI]

[16] Grant SF, Hakonarson H. Genome-wide association studies in type 1 diabetes. Curr Diab Rep, 2009, 9(2): 157-163. [DOI]

[17] Grant SF, Thorleifsson G, Reynisdottir I, Benediktsson R, Manolescu A, Sainz J, Helgason A, Stefansson H, Emilsson V, Helgadottir A, Styrkarsdottir U, Magnusson KP, Walters GB, Palsdottir E, Jonsdottir T, Gudmundsdottir T, Gylfason A, Saemundsdottir J, Wilensky RL, Reilly MP, Rader DJ, Bagger Y, Christiansen C, Gudnason V, Sigurdsson G, Thorsteinsdottir U, Gulcher JR, Kong A, Stefansson K. Variant of transcription factor 7-like 2 (TCF7L2) gene confers risk of type 2 diabetes. Nat Genet, 2006, 38(3): 320-323. [DOI]

[18] Cauchi S, El Achhab Y, Choquet H, Dina C, Krempler F, Weitgasser R, Nejjari C, Patsch W, Chikri M, Meyre D, Froguel P. TCF7L2 is reproducibly associated with type 2 diabetes in various ethnic groups: a global meta-analysis. $J$ Mol Med, 2007, 85(7): 777-782. [DOI]

[19] Sladek R, Rocheleau G, Rung J, Dina C, Shen L, Serre D, Boutin P, Vincent D, Belisle A, Hadjadj S, Balkau B, Heude B, Charpentier G, Hudson TJ, Montpetit A, Pshezhetsky AV, Prentki M, Posner BI, Balding DJ, Meyre D, Polychronakos C, Froguel P. A genome-wide association study identifies novel risk loci for type 2 diabetes. Nature, 2007, 445(7130): 881-885. [DOI]

[20] Salonen JT, Uimari P, Aalto JM, Pirskanen M, Kaikkonen J, Todorova B, Hypponen J, Korhonen VP, Asikainen J, Devine C, Tuomainen TP, Luedemann J, Nauck M, Kerner W, Stephens RH, New JP, Ollier WE, Gibson JM, Payton A, Horan MA, Pendleton N, Mahoney W, Meyre D, Delplanque J, Froguel P, Luzzatto O, Yakir B, Darvasi A. Type 2 diabetes whole-genome association study in four populations: the DiaGen consortium. Am J Hum Genet, 2007, 81(2): 338-345. [DOI]

[21] Steinthorsdottir V, Thorleifsson G, Reynisdottir I, Benediktsson R, Jonsdottir T, Walters GB, Styrkarsdottir U, Gretarsdottir S, Emilsson V, Ghosh S, Baker A, Snorradottir S, Bjarnason H, Ng MC, Hansen T, Bagger Y, Wilensky RL, Reilly MP, Adeyemo A, Chen Y, Zhou J, Gudnason V, Chen G, Huang H, Lashley K, Doumatey A, So WY, Ma $\mathrm{RC}$, Andersen G, Borch-Johnsen K, Jorgensen T, van Vliet-Ostaptchouk JV, Hofker MH, Wijmenga C,
Christiansen C, Rader DJ, Rotimi C, Gurney M, Chan JC, Pedersen O, Sigurdsson G, Gulcher JR, Thorsteinsdottir U, Kong A, Stefansson K. A variant in CDKAL1 influences insulin response and risk of type 2 diabetes. Nat Genet, 2007, 39(6): 770-775. [DOI]

[22] Scott LJ, Mohlke KL, Bonnycastle LL, Willer CJ, Li Y, Duren WL, Erdos MR, Stringham HM, Chines PS, Jackson AU, Prokunina-Olsson L, Ding CJ, Swift AJ, Narisu N, Hu TL, Pruim R, Xiao R, Li XY, Conneely KN, Riebow NL, Sprau AG, Tong M, White PP, Hetrick KN, Barnhart MW, Bark CW, Goldstein JL, Watkins L, Xiang F, Saramies J, Buchanan TA, Watanabe RM, Valle TT, Kinnunen L, Abecasis GR, Pugh EW, Doheny KF, Bergman RN, Tuomilehto J, Collins FS, Boehnke M. A genome-wide association study of type 2 diabetes in Finns detects multiple susceptibility variants. Science, 2007, 316(5829): 1341-1345. [DOI]

[23] McCarthy MI, Zeggini E. Genome-wide association studies in type 2 diabetes. Curr Diab Rep, 2009, 9(2): 164-171. [DOI]

[24] Mueller PW. Technologies for diabetes genomics. J Diabetes Sci Technol, 2009, 3(4): 735-738. [DOI]

[25] Hirschhorn JN, Lettre G. Progress in genome-wide association studies of human height. Horm Res, 2009, 71(Suppl 2): 5-13. [DOI]

[26] Loos RJ. Recent progress in the genetics of common obesity. Br J Clin Pharmacol, 2009, 68(6): 811-829. [DOI]

[27] Tai ES, Ordovas JM. Genome-wide association studies for blood lipids. A great start but a long way to go. Curr Opin Lipidol, 2010, 21(2): 101-103. [DOI]

[28] Sturm RA. Molecular genetics of human pigmentation diversity. Hum Mol Genet, 2009, 18(R1): R9-R17. [DOI]

[29] Choi HK, Zhu Y, Mount DB. Genetics of gout. Curr Opin Rheumatol, 2010, 22(2): 144-151. [DOI]

[30] Ferrari S. Human genetics of osteoporosis. Best Pract Res Clin Endocrinol Metab, 2008, 22(5): 723-735. [DOI]

[31] Weiss ST. Lung function and airway diseases. Nat Genet, 2010, 42(1): 14-16. [DOI]

[32] Benjamin EJ, Dupuis J, Larson MG, Lunetta KL, Booth SL, Govindaraju DR, Kathiresan S, Keaney JF, Jr., Keyes MJ, Lin JP, Meigs JB, Robins SJ, Rong J, Schnabel R, Vita JA, Wang TJ, Wilson PW, Wolf PA, Vasan RS. Genome-wide association with select biomarker traits in the Framingham Heart Study. BMC Med Genet, 2007, 8(Suppl 1): S11. [DOI] 
[33] Frayling TM, Timpson NJ, Weedon MN, Zeggini E, Freathy RM, Lindgren CM, Perry JR, Elliott KS, Lango H, Rayner NW, Shields B, Harries LW, Barrett JC, Ellard S, Groves CJ, Knight B, Patch AM, Ness AR, Ebrahim S, Lawlor DA, Ring SM, Ben-Shlomo Y, Jarvelin MR, Sovio U, Bennett AJ, Melzer D, Ferrucci L, Loos RJ, Barroso I, Wareham NJ, Karpe F, Owen KR, Cardon LR, Walker M, Hitman GA, Palmer CN, Doney AS, Morris AD, Smith GD, Hattersley AT, McCarthy MI. A common variant in the FTO gene is associated with body mass index and predisposes to childhood and adult obesity. Science, 2007, 316(5826): 889-894. [DOI]

[34] Scuteri A, Sanna S, Chen WM, Uda M, Albai G, Strait J, Najjar S, Nagaraja R, Orrú M, Usala G, Dei M, Lai S, Maschio A, Busonero F, Mulas A, Ehret GB, Fink AA, Weder AB, Cooper RS, Galan P, Chakravarti A, Schlessinger D, Cao A, Lakatta E, Abecasis GR. Genome-wide association scan shows genetic variants in the FTO gene are associated with obesity-related traits. PLoS Genet, 2007, 3(7): e115. [DOI]

[35] Han J, Kraft P, Nan H, Guo Q, Chen C, Qureshi A, Hankinson SE, Hu FB, Duffy DL, Zhao ZZ, Martin NG, Montgomery GW, Hayward NK, Thomas G, Hoover RN, Chanock S, Hunter DJ. A genome-wide association study identifies novel alleles associated with hair color and skin pigmentation. PLoS Genet, 2008, 4(5): e1000074. [DOI]

[36] Bishop DT, Demenais F, Iles MM, Harland M, Taylor JC, Corda E, Randerson-Moor J, Aitken JF, Avril MF, Azizi E, Bakker B, Bianchi-Scarrá G, Bressac-de Paillerets B, Calista D, Cannon-Albright LA, Chin AWT, Debniak T, Galore-Haskel G, Ghiorzo P, Gut I, Hansson J, Hocevar M, Höiom V, Hopper JL, Ingvar C, Kanetsky PA, Kefford RF, Landi MT, Lang J, Lubinski J, Mackie R, Malvehy J, Mann GJ, Martin NG, Montgomery GW, van Nieuwpoort FA, Novakovic S, Olsson H, Puig S, Weiss M, van Workum W, Zelenika D, Brown KM, Goldstein AM, Gillanders EM, Boland A, Galan P, Elder DE, Gruis NA, Hayward NK, Lathrop GM, Barrett JH, Bishop JA. Genome-wide association study identifies three loci associated with melanoma risk. Nat Genet, 2009, 41(8): 920-925. [DOI]

[37] Jin Y, Birlea SA, Fain PR, Gowan K, Riccardi SL, Holland PJ, Mailloux CM, Sufit AJ, Hutton SM, Amadi-Myers A, Bennett DC, Wallace MR, McCormack WT, Kemp EH, Gawkrodger DJ, Weetman AP, Picardo M, Leone G, Taïeb A, Jouary T, Ezzedine K, van Geel N, Lambert J, Overbeck
A, Spritz RA. Variant of TYR and autoimmunity susceptibility loci in generalized vitiligo. $N$ Engl J Med, 2010, 362(18): 1686-1697. [DOI]

[38] Gudbjartsson DF, Sulem P, Stacey SN, Goldstein AM, Rafnar T, Sigurgeirsson B, Benediktsdottir KR, Thorisdottir K, Ragnarsson R, Sveinsdottir SG, Magnusson V, Lindblom A, Kostulas K, Botella-Estrada R, Soriano V, Juberias P, Grasa M, Saez B, Andres R, Scherer D, Rudnai P, Gurzau E, Koppova K, Kiemeney LA, Jakobsdottir M, Steinberg S, Helgason A, Gretarsdottir S, Tucker MA, Mayordomo JI, Nagore E, Kumar R, Hansson J, Olafsson JH, Gulcher J, Kong A, Thorsteinsdottir U, Stefansson K. ASIP and TYR pigmentation variants associate with cutaneous melanoma and basal cell carcinoma. Nat Genet, 2008, 40(7): 886-891. [DOI]

[39] Need AC, Goldstein DB. Whole genome association studies in complex diseases: where do we stand? Dialogues Clin Neurosci, 2010, 12(1): 37-46. [DOI]

[40] Daly AK. Pharmacogenetics and human genetic polymorphisms. Biochem J, 2010, 429(3): 435-449. [DOI]

[41] O'Donovan MC, Craddock N, Norton N, Williams H, Peirce T, Moskvina V, Nikolov I, Hamshere M, Carroll L, Georgieva L, Dwyer S, Holmans P, Marchini JL, Spencer CC, Howie B, Leung HT, Hartmann AM, Möller HJ, Morris DW, Shi Y, Feng G, Hoffmann P, Propping P, Vasilescu C, Maier W, Rietschel M, Zammit S, Schumacher J, Quinn EM, Schulze TG, Williams NM, Giegling I, Iwata N, Ikeda M, Darvasi A, Shifman S, He L, Duan J, Sanders AR, Levinson DF, Gejman PV, Cichon S, Nöthen MM, Gill M, Corvin A, Rujescu D, Kirov G, Owen MJ, Buccola NG, Mowry BJ, Freedman R, Amin F, Black DW, Silverman JM, Byerley WF, Cloninger CR. Identification of loci associated with schizophrenia by genome-wide association and follow-up. Nat Genet, 2008, 40(9): 1053-1055. [DOI]

[42] de Cid R, Riveira-Munoz E, Zeeuwen PL, Robarge J, Liao W, Dannhauser EN, Giardina E, Stuart PE, Nair R, Helms C, Escaramí s G, Ballana E, Martin-Ezquerra G, den Heijer M, Kamsteeg M, Joosten I, Eichler EE, Lazaro C, Pujol RM, Armengol L, Abecasis G, Elder JT, Novelli G, Armour JA, Kwok PY, Bowcock A, Schalkwijk J, Estivill X. Deletion of the late cornified envelope $L C E 3 B$ and $L C E 3 C$ genes as a susceptibility factor for psoriasis. Nat Genet, 2009, 41(2): 211-215. [DOI]

[43] Zhang XJ, Huang W, Yang S, Sun LD, Zhang FY, Zhu QX, Zhang FR, Zhang C, Du WH, Pu XM, Li H, Xiao FL, 
Wang ZX, Cui Y, Hao F, Zheng J, Yang XQ, Cheng H, He CD, Liu XM, Xu LM, Zheng HF, Zhang SM, Zhang JZ, Wang HY, Cheng YL, Ji BH, Fang QY, Li YZ, Zhou FS, Han JW, Quan C, Chen B, Liu JL, Lin D, Fan L, Zhang AP, Liu SX, Yang CJ, Wang PG, Zhou WM, Lin GS, Wu WD, Fan X, Gao M, Yang BQ, Lu WS, Zhang Z, Zhu KJ, Shen SK, Li M, Zhang XY, Cao TT, Ren W, Zhang X, He J, Tang XF, Lu S, Yang JQ, Zhang L, Wang DN, Yuan F, Yin XY, Huang HJ, Wang HF, Lin XY, Liu JJ. Psoriasis genome-wide association study identifies susceptibility variants within LCE gene cluster at 1q21. Nat Genet, 2009, 41(2): 205-210. [DOI]

[44] Han JW, Zheng HF, Cui Y, Sun LD, Ye DQ, Hu Z, Xu JH, Cai ZM, Huang W, Zhao GP, Xie HF, Fang H, Lu QJ, Xu JH, Li XP, Pan YF, Deng DQ, Zeng FQ, Ye ZZ, Zhang XY, Wang QW, Hao F, Ma L, Zuo XB, Zhou FS, Du WH, Cheng YL, Yang JQ, Shen SK, Li J, Sheng YJ, Zuo XX, Zhu WF, Gao F, Zhang PL, Guo Q, Li B, Gao M, Xiao FL, Quan C, Zhang C, Zhang Z, Zhu KJ, Li Y, Hu DY, Lu WS, Huang JL, Liu SX, Li H, Ren YQ, Wang ZX, Yang CJ, Wang PG, Zhou WM, Lv YM, Zhang AP, Zhang SQ, Lin D, Li Y, Low HQ, Shen M, Zhai ZF, Wang Y, Zhang FY, Yang S, Liu JJ, Zhang XJ. Genome-wide association study in a Chinese Han population identifies nine new susceptibility loci for systemic lupus erythematosus. Nat Genet, 2009, 41(11): 1234-1237. [DOI]

[45] Gateva V, Sandling JK, Hom G, Taylor KE, Chung SA, Sun X, Ortmann W, Kosoy R, Ferreira RC, Nordmark G, Gunnarsson I, Svenungsson E, Padyukov L, Sturfelt G, Jonsen A, Bengtsson AA, Rantapää-Dahlqvist S, Baechler EC, Brown EE, Alarcon GS, Edberg JC, Ramsey-Goldman R, McGwin G, Jr., Reveille JD, Vila LM, Kimberly RP, Manzi S, Petri MA, Lee A, Gregersen PK, Seldin MF, Rönnblom L, Criswell LA, Syvanen AC, Behrens TW, Graham RR. A large-scale replication study identifies TNIP1, PRDM1, JAZF1, UHRF1BP1 and IL10 as risk loci for systemic lupus erythematosus. Nat Genet, 2009, 41(11): 1228-1233. [DOI]

[46] Yang W, Shen N, Ye DQ, Liu Q, Zhang Y, Qian XX, Hirankarn N, Ying D, Pan HF, Mok CC, Chan TM, Wong RW, Lee KW, Mok MY, Wong SN, Leung AM, Li XP, Avihingsanon Y, Wong CM, Lee TL, Ho MH, Lee PP, Chang YK, Li PH, Li RJ, Zhang L, Wong WH, Ng IO, Lau CS, Sham PC, Lau YL. Genome-wide association study in Asian populations identifies variants in ETS1 and WDFY4 associated with systemic lupus erythematosus. PLoS Genet,
2010, 6(2): e1000841. [DOI]

[47] Zhang FR, Huang W, Chen SM, Sun LD, Liu H, Li Y, Cui Y, Yan XX, Yang HT, Yang RD, Chu TS, Zhang C, Zhang L, Han JW, Yu GQ, Quan C, Yu YX, Zhang Z, Shi BQ, Zhang LH, Cheng H, Wang CY, Lin Y, Zheng HF, Fu XA, Zuo XB, Wang Q, Long H, Sun YP, Cheng YL, Tian HQ, Zhou FS, Liu HX, Lu WS, He SM, Du WL, Shen M, Jin QY, Wang Y, Low HQ, Erwin T, Yang NH, Li JY, Zhao X, Jiao YL, Mao LG, Yin G, Jiang ZX, Wang XD, Yu JP, Hu ZH, Gong CH, Liu YQ, Liu RY, Wang DM, Wei D, Liu JX, Cao WK, Cao HZ, Li YP, Yan WG, Wei SY, Wang KJ, Hibberd ML, Yang S, Zhang XJ, Liu JJ. Genomewide association study of leprosy. N Engl J Med, 2009, 361(27): 2609-2618. [DOI]

[48] Le Bourhis L, Benko S, Girardin SE. Nod1 and Nod2 in innate immunity and human inflammatory disorders. Biochem Soc Trans, 2007, 35(Pt 6): 1479-1484. [DOI]

[49] Barrett JC, Hansoul S, Nicolae DL, Cho JH, Duerr RH, Rioux JD, Brant SR, Silverberg MS, Taylor KD, Barmada MM, Bitton A, Dassopoulos T, Datta LW, Green T, Griffiths AM, Kistner EO, Murtha MT, Regueiro MD, Rotter JI, Schumm LP, Steinhart AH, Targan SR, Xavier RJ, Libioulle C, Sandor C, Lathrop M, Belaiche J, Dewit O, Gut I, Heath S, Laukens D, Mni M, Rutgeerts P, Van Gossum A, Zelenika D, Franchimont D, Hugot JP, de Vos M, Vermeire S, Louis E, Cardon LR, Anderson CA, Drummond H, Nimmo E, Ahmad T, Prescott NJ, Onnie CM, Fisher SA, Marchini J, Ghori J, Bumpstead S, Gwilliam R, Tremelling M, Deloukas P, Mansfield J, Jewell D, Satsangi J, Mathew CG, Parkes M, Georges M, Daly MJ. Genome-wide association defines more than 30 distinct susceptibility loci for Crohn's disease. Nat Genet, 2008, 40(8): 955-962. [DOI]

[50] Quan C, Ren YQ, Xiang LH, Sun LD, Xu AE, Gao XH, Chen HD, Pu XM, Wu RN, Liang CZ, Li JB, Gao TW, Zhang JZ, Wang XL, Wang J, Yang RY, Liang L, Yu JB, Zuo XB, Zhang SQ, Zhang SM, Chen G, Zheng XD, Li P, Zhu J, Li YW, Wei XD, Hong WS, Ye Y, Zhang Y, Wu WS, Cheng H, Dong PL, Hu DY, Li Y, Li M, Zhang X, Tang HY, Tang XF, Xu SX, He SM, Lv YM, Shen M, Jiang HQ, Wang Y, Li K, Kang XJ, Liu YQ, Sun L, Liu ZF, Xie SQ, Zhu CY, Xu Q, Gao JP, Hu WL, Ni C, Pan TM, Yao S, He CF, Liu YS, Yu ZY, Yin XY, Zhang FY, Yang S, Zhou Y, Zhang XJ. Genome-wide association study for vitiligo identifies susceptibility loci at $6 \mathrm{q} 27$ and the MHC. Nat Genet, 2010, 42(7): 614-618. [DOI]

[51] Bei JX, Li Y, Jia WH, Feng BJ, Zhou G, Chen LZ, Feng QS, 
Low HQ, Zhang H, He F, Tai ES, Kang T, Liu ET, Liu J, Zeng YX. A genome-wide association study of nasopharyngeal carcinoma identifies three new susceptibility loci. Nat Genet, 2010, 42(7): 599-603. [DOI]

[52] Zhang HX, Zhai Y, Hu ZB, Wu C, Qian J, Jia WH, Ma FC, Huang WF, Yu LX, Yue W, Wang ZF, Li PY, Zhang Y, Liang RX, Wei ZL, Cui Y, Xie WM, Cai M, Yu XS, Yuan YF, Xia X, Zhang XM, Yang H, Qiu W, Yang JM, Gong F, Chen MS, Shen HB, Lin DX, Zeng YX, He FC, Zhou GQ. Genome-wide association study identifies 1 p36.22 as a new susceptibility locus for hepatocellular carcinoma in chronic hepatitis B virus carriers. Nat Genet, 2010, 42(9): 755-758. [DOI]

[53] Wang LD, Zhou FY, Li XM, Sun LD, Song X, Jin Y, Li JM, Kong GQ, Qi H, Cui J, Zhang LQ, Yang JZ, Li JL, Li XC, Ren JL, Liu ZC, Gao WJ, Yuan L, Wei W, Zhang YR, Wang WP, Sheyhidin I, Li F, Chen BP, Ren SW, Liu B, Li D, Ku JW, Fan ZM, Zhou SL, Guo ZG, Zhao XK, Liu N, Ai YH, Shen FF, Cui WY, Song S, Guo T, Huang J, Yuan C, Wu Y, Yue WB, Feng CW, Li HL, Wang Y, Tian JY, Lu Y, Yuan Y, Zhu WL, Liu M, Fu WJ, Yang X, Wang HJ, Han SL, Chen J, Han M, Wang HY, Zhang P, Dong JC, Xing GL, Wang R, Guo M, Chang ZW, Liu HL, Guo L, Yuan ZQ, Liu H, Lu Q, Yang LQ, Zhu FG, Yang XF, Feng XS, Wang Z, Li Y, Gao SG, Qige Q, Bai LT, Yang WJ, Lei GY, Shen ZY,
Chen LQ, Li EM, Xu LY, Wu ZY, Cao WK, Wang JP, Bao ZQ, Chen JL, Ding GC, Zhuang X, Zhou YF, Zheng HF, Zhang Z, Zuo XB, Dong ZM, Fan DM, He X, Wang J, Zhou Q, Zhang QX, Jiao XY, Lian SY, Ji AF, Lu XM, Wang JS, Chang FB, Lu CD, Chen ZG, Miao JJ, Fan ZL, Lin RB, Liu TJ, Wei JC, Kong QP, Lan Y, Fan YJ, Gao FS, Wang TY, Xie D, Chen SQ, Yang WC, Hong JY, Wang L, Qiu SL, Cai ZM, Zhang XJ. Genome-wide association study of esophageal squamous cell carcinoma in Chinese subjects identifies susceptibility loci at PLCE1 and C20orf54. Nat Genet, 2010, 42(9): 759-763. [DOI]

[54] Sun LD, Cheng H, Wang ZX, Zhang AP, Wang PG, Xu JH, Zhu QX, Zhou HS, Ellinghaus E, Zhang FR, Pu XM, Yang XQ, Zhang JZ, Xu AE, Wu RN, Xu LM, Peng L, Helms CA, Ren YQ, Zhang C, Zhang SM, Nair RP, Wang HY, Lin GS, Stuart PE, Fan X, Chen G, Tejasvi T, Li P, Zhu J, Li ZM, Ge HM, Weichenthal M, Ye WZ, Zhang C, Shen SK, Yang BQ, Sun YY, Li SS, Lin Y, Jiang JH, Li CT, Chen RX, Cheng J, Jiang X, Zhang P, Song WM, Tang J, Zhang HQ, Sun L, Cui J, Zhang LJ, Tang B, Huang F, Qin Q, Pei XP, Zhou AM, Shao LM, Liu JL, Zhang FY, Du WD, Franke A, Bowcock AM, Elder JT, Liu JJ, Yang S, Zhang XJ. Association analyses identify six new psoriasis susceptibility loci in the Chinese population. Nat Genet. 2010, 42(11): 1005-1009. [DOI] 